\title{
Update on antibacterial agents: current challenges and recent initiatives
}

\author{
G.P.S.G. Senadeera ${ }^{1}$, S. Sri Ranganathan ${ }^{2}$ \\ ${ }^{1}$ Department of Allied Health Sciences, Faculty of Medical Sciences, University of Sri Jayewardenepura \\ ${ }^{2}$ Department of Pharmacology, Faculty of Medicine, University of Colombo, Sri Lanka
}

\begin{abstract}
Antibacterial agents (ABAs) contribute significantly to reduce morbidity and mortality of bacterial infections as well as play a crucial role in the success of major advances in medicine such as organ transplants, advanced surgeries, cancer chemotherapy and cardiac surgery. However, their success as well as their very existence itself are under threat due to two major problems, one is antibacterial resistance $(\mathrm{ABR})$ and the other is discovery void.
\end{abstract}

A 2014 report by the World Health Organization (WHO) warns that bacteria that cause common health-care associated and community-acquired infections exhibit high resistance rate in all WHO regions. This threat has been endorsed by many organizations including Centres for Disease Control and Prevention (CDC), Infectious Diseases Society of America, and other UN bodies. Though bacteria can develop resistance spontaneously through mutation, the escalating public health threat of ABR is mainly driven by both appropriate and inappropriate use of ABAs in humans, animals, food production, agriculture, and aquaculture. Several initiatives at different levels have been launched to combatABR.

Development of new ABAs which feature new target or mode of action by pharmaceutical industries has the potential to address the problem of ABR. However, hardly any new ABAs featuring new target or mode of action came to market in the last two decades due to economic and regulatory obstacles. Collaboration between industry, government bodies and academic institutions in the exploration of new ABAs, offering incentives, fast tracking market authorization are some of the initiatives recommended by the WHO to address this issue of dry antibiotic pipeline. Rational use of ABAs, implementation of antibiotic stewardship programmes, and adherence to strategies which minimise spread of resistant bacteria such as hand-washing and infection control measures are few key activities that can be incorporated in clinical practice.

Correspondence: S. Sri Ranganathan
E-mail: sshalini14@hotmail.com
Received: 04-11-2017 Accepted: 22-12-2017
(D) http://orcid.org/0000-0003-0184-104X CC C
DOI: http://doi.org/10.4038/sljs.v35i4.8435

The Sri Lanka Journal of Surgery 2017; 35(4): 23-28

\section{Introduction}

Antibacterial agents (ABA) have saved millions of lives. Not only do they contribute significantly to reduce morbidity and mortality of individual classic infectious diseases, but also serve a crucial role in the success of modern medicine such as advanced surgical procedures, organ transplants, cancer chemotherapy, neonatal care and intensive-care. However, the achievement of ABAs is currently under threat as antibacterial resistance (ABR) is escalating at an exponential rate. This review presents an overview of current trends of ABR and few key global and national initiatives towards combating ABR.

\section{ACCESS, WATCH and RESERVE antibacterial agents}

The World Health Organization (WHO) recently revised the entire antibacterial agents section in the model essential medicine list (EML) [1]. This was the biggest revision in the 40- year history of WHO Model EML. The WHO Expert panel on selection and use of essential medicines has grouped the ABAs into three categories namely ACCESS, WATCH and RESERVE which is accompanied by recommendations on indications for each category. The list encompasses ABAs for 21 most common general infections which includes urinary tract infections, skin and soft tissue infections, surgical site infections, hospital acquired pneumonia, complicated intra-abdominal infections and bone and joint infections. It is postulated that this categorization will ensure access to ABAs when needed, rational prescription of right ABAs for right infections, improve treatment outcomes, reduce the development of drug-resistant bacteria, and conserve the effectiveness of "last-resort" ABAs that are needed when all others fail. Comprehending the rationale for this categorization is important to use this categorization in clinical practice and policy making.

Antibacterial agents which are either first or second choice in at least one of the above 21 syndromes are classified as ACCESS ABAs (Table 1) [1].

"These essential ABAs are those that satisfy the priority health care needs of the population and intended to be available within the context of functioning health systems at all times in adequate amounts, in the appropriate dosage 
Table 1. Antibacterial agents listed in ACCESS group

\begin{tabular}{|l|l|l|l|}
\hline \multicolumn{2}{|c|}{ Beta-lactam medicines } & \multicolumn{2}{c|}{ Other antibacterial } \\
\hline amoxicillin & cefotaxime* & amikacin & gentamicin \\
\hline amoxicillin + clavulanic acid & ceftriaxone* & azithromycin* & metronidazole \\
\hline ampicillin & cloxacillin & chloramphenicol & nitrofurantoin \\
\hline benzathine benzylpenicillin & Phenoxymethyl penicillin & ciprofloxacin* & Spectinomycin (EML only) \\
\hline benzylpenicillin & piperacillin + tazobactam** & clarithromycin* & sulfamethoxazole + trimethoprim \\
\hline cefalexin & procaine benzyl penicillin & clindamycin & vancomycin (oral)* \\
\hline cefazolin & meropenem* & doxycycline & voncomycin (parenteral) \\
\hline
\end{tabular}

Italics $=$ complementary list in the essential medicine list

*Watch group antibiotics included in the essential medicine list only for specific, limited indications

Table 2. Antibacterial agents listed in WATCH group

\begin{tabular}{|l|}
\hline $\begin{array}{l}\text { Quinolones and fluoroquinolones: } \\
\text { e.g. ciprofloxacin, levofloxacin, moxifloxacin, norfloxacin }\end{array}$ \\
\hline (wrd-generation cephalosporins \\
e.g. cefixime, ceftriaxone, cefotaxime, ceftazidime \\
\hline Macrolides: e.g. azithromycin, clarithromycin, erythromycin \\
\hline Glycopeptides: e.g. teicoplanin, vancomycin \\
\hline Anti-pseudomonal penicillins with beta-lactamase inhibitor: \\
e.g. piperacillin + tazobactam \\
\hline Carbapenems: e.g. meropenem, imipenem + cilastatin \\
\hline Penems: e.g. faropenem
\end{tabular}

Table 3. Antibacterial agents listed in RESERVE (last-resort) group

\begin{tabular}{|l|l|}
\hline Aztreonam & Fosfomycin (IV) \\
\hline $\begin{array}{l}\text { 4th generation cephalosporins } \\
\text { e.g. cefepime }\end{array}$ & $\begin{array}{l}\text { Oxazolidinones } \\
\text { e.g. linezolid }\end{array}$ \\
\hline $\begin{array}{l}\text { 5th generation cephalosporins } \\
\text { e.g. ceftaroline }\end{array}$ & Tigecycline \\
\hline $\begin{array}{l}\text { Polymyxins } \\
\text { e.g. polymyxin B, colistin }\end{array}$ & Daptomycin \\
\hline
\end{tabular}

forms, with assured quality, and at a price the individual and the community can afford. They are selected on the basis of disease prevalence, evidence on efficacy, safety and comparative cost-effectiveness [2]".

The main function of the WHO EML expert committee is to update the WHO model EML which is done every 2 years. In previous years, the committee stopped with selection of essential ABAs (ACCESS group). The 2017 committee extended its role and categorized the remaining ABAs into two groups mainly to assist ABA stewardship programmes.
The "WATCH" group includes ABA classes that are recommended as first or second choice treatments for a limited number of indications, but are flagged as having high resistance potential (Table 2). The RESERVE group identifies ABAs that should be considered as last-resort when other alternatives would be inadequate or have already failed (e.g., serious life-threatening infections due to multi-drug resistant bacteria). They are expected to be personalized to highly specific patients and settings (Table 3). Understandably, there are some overlaps: For example, some ABAs in the ACCESS group are listed under WATCH group as well since there are concerns about resistance. Their use should be monitored.

The message delivered is: Ensure "universal access" to first group, "watch" the use of second group and "reserve" the third group as much as possible. Considerable amount of background work has been done by a group of global experts on adult and paediatric infectious diseases as well as by the members of the WHO expert committee representing both resource limited and rich countries in formulating this categorization. It is highly recommended that the healthcare professionals, administrators and policy makers give serious attention to this recommendation if interested in combating antibacterial resistance (ABR). This aim of this classification is to guide rational choice of ABAs and not function as guidelines. The expert committee stressed that the treatment decisions will depend on local/national uniqueness including availability of ABAs and local resistance patterns; hence clinical judgment would be the decisive factor when it comes to treatment of an individual patient.

\section{National Guidelines for empirical and prophylactic use of antimicrobials}

In 2016, the Sri Lanka College of Microbiologists in collaboration with the other professional colleges in healthcare and the Ministry of Healthcare and Nutrition published the National Guidelines for the empirical and prophylactic use of antimicrobials [3]. Twenty Professional Colleges and Associations including the College of Surgeons have contributed in developing the guidelines. It gives the 
national recommendations for 22 infections which require ABAs. Examples include bacterial endocarditis, bone and joint infections, intra-abdominal infections, surgicalprophylaxis, urinary tract infections in adults, childhood urinary tract infections and trauma prophylaxis. Each infection is further categorized and recommendations for primary and alternative therapy are listed. Details of agent, dose and route of administration are indicated. In addition, relevant comments for example, investigations to be done, decision about duration of therapy, important precautions, etc can be found.

Any guidelines on use of antimicrobials cannot give definitive recommendation because ABAs had to act against living organisms. Virulence and resistance of these living organisms dictate the final decision which differs from patient to patient, setting to setting and time to time. The Sri Lankan guidelines clearly indicate that the ABA should be tailored whenever a microbiological diagnosis and antibiotic susceptibility results are available. The guidelines will be a very useful guide for a clinician, especially when rapid microbiological diagnostic facilities are not available. However, it will not substitute clinical acumen and hospital level data on antibacterial sensitivity. In addition, treating an infection successfully goes beyond the boundaries of selecting the correct ABA. Pharmacokinetics and dynamics play key role in successful treatment of an infection [4]. Hence, rational use of ABAs and adherence to other strategies which combat ABR such as hand-washing, infection control, etc. are equally important. The guidelines is available for free downloading in the College of Microbiologists website (slmicrobiology.net/download/National-AntibioticGuidelines-2016-Web.pdf)

\section{Surgical prophylaxis and surgical-site infection (SSI)}

Guidelines for surgical prophylaxis are available in his National Guidelines. Surgical procedures have been classified clean, potentially contaminate (clean contaminated), contaminated and dirty (infected) as per the National Institute for Health and Care Excellence-UK (NICE-UK, 2008) classification [5]. Antibacterial prophylaxis is indicated in some clean (e.g.; surgery involving introduction of prosthetic material, surgery where consequences of infection would be catastrophic like open heart surgery and surgery with impaired host defence) and all clean-contaminated surgeries. For contaminated surgeries either ABA prophylaxis or therapy is recommended depending on patient's clinical condition and for dirty surgeries $\mathrm{ABA}$ therapy is indicated. It also outlines patient care recommendations, and recommended agent/s. dose, duration and timing of ABA prophylaxis. Recently both WHO and Centres for Disease Control and Prevention (CDC) have published extensive stand-alone guidelines on surgical - site infections $[6,7]$.

\section{Non human-use of antibacterial agents}

Use of ABAs in veterinary medicine, food-animal industry, livestock production (mainly poultry, cattle, turkeys and pigs), agriculture and aquaculture contributes significantly to development and spread of ABR [8-10]. Infections caused by these resistant bacteria are transmitted to humans via foodchain, contaminated hands and environment.

In USA, it is estimated that about 1 in 5 resistant infections are caused by germs from food and animals [11]. Polypeptides, macrolides, quinolones and penicillins are approved for nontherapeutic use (growth promotion) in animal agriculture. Fish-farming industry uses ABAs to prevent disease, promote growth, increase yield and treat infections. In animal husbandry, ABAs are often used in sub-therapeutic doses for a longer period of time. When few animals are affected by an infection, the entire group is treated with ABA irrespective of whether they are sick or healthy [8-10]. Already, European Union has imposed a ban on feeding antibiotics to livestock for growth promotion [9].

In this context, the recently published 5 th revision of critically important antimicrobials (CIA) for human medicine by the World Health Organization Advisory Group on Integrated Surveillance of Antimicrobial Resistance [12] is a crucial initiative. It is a collaborative effort of Food and Agriculture Organization of the United Nations (FAO), the World Organization for Animal Health (OIE), and World Health Organization (WHO). Key object of this list is to rank medically important antimicrobials for risk management of antimicrobial resistance due to non-human use.

The list classifies ABAs into three namely [1] critically important, [2] highly important and [3] important for human medicine. Antimicrobials listed under critically important group are the "sole or one of limited available therapies, to treat serious bacterial infections in people" and "used to treat infections in people caused by either: [1] bacteria that may be transmitted to humans from non-human sources, or [2] bacteria that may acquire resistance genes from non-human sources". Example includes cephalosporin (3rd, 4th and 5th generation), glycopeptides, macrolides, ketolides, polymyxins and quinolones.

Recently, the National Strategic Plan for combating antimicrobial resistance (2017-2022) was launched in Sri Lanka as a collaborative programme of Ministries of Health, Fisheries and Aquatic Resources Management, Agriculture and Rural Economy with the support and concurrence of the World Health Organization [13]. There are five strategies [1] Improve awareness and understanding of antimicrobial resistance through effective communication [2] strengthen the knowledge and evidence base surveillance and research [3] reduce the incidence of infection through effective sanitation, hygiene and infection prevention measures [4] 
optimize the use of antimicrobial medicines in human and animal health [5] prepare the economic case for sustainable investment and increase investment in new medicines, diagnostic tools, vaccines and other interventions. Each strategy has specific objectives and mile-stones to achieve.

\section{Integrated surveillance of antimicrobial resistance and use}

Surveillance of ABR and ABA use is an essential component to contain ABR [14]. The European Surveillance of Antimicrobial Consumption network (ESAC-Net) has demonstrated that monitoring antimicrobial use patterns and costs is an important resource for advocacy at local or national level, especially when surveillance of use is enhanced by surveillance of resistance [15]. Three surveillance programmes in Sri Lanka need special mention.

\section{Surveillance of antimicrobial resistance}

In 2011, the Sri Lanka College of Microbiologists, in collaboration with the Ministry of Healthcare initiated the National Laboratory Based Surveillance of Antimicrobial Resistance. The goal was to survey the pooled susceptibility of significant isolates (colony count $\geq 105 \mathrm{CFU} / \mathrm{ml}$ ) cultured from urine of non-catheterized patients. In 2014, data from seven centres showed that the majority $(80 \%)$ of isolates were coliforms with a very high resistance rate for ampicillin $(90.1 \%)$.

Those isolated from adults attending outpatient clinics were most susceptible to meropenem $(100 \%)$ followed by imipenam (97.4\%), gentamicin $(86.4 \%)$, nitrofurantoin (65.1\%), cefota-xime (63.4\%), cephalexin (55.2\%), cephradine (55.2\%), amoxicillin/clavulanic acid (54\%) and norfloxacin $(48.3 \%)$. Those isolated from adult hospitalized patients were most susceptible to meropenem $(87.9 \%)$ followed by gentamicin $(62.6 \%)$, cefotaxime $(39.5 \%)$ and ciprofloxacin $(31.9 \%)$. Resistance rate was comparatively less with coliforms isolated from paediatric out-patients [16].

Secondly, in 2013, the Sri Lanka College of Microbiologists reported the results of Antimicrobial Resistance Surveillance Project (ARSP) of Phase 1.

This surveillance programme exa-mined the Gram negative bacteria and their susceptibility to ABAs in patients who were clinically managed as bacterae-mia in seven hospitals during 2009-2010 period: Of the 733 Gram negative isolates studied, ESBL producing Escherichia coli and Klebsiella pneumonia accounted for $22.5 \%$.

They were most susceptible to meropenem and imipenam (100\%) followed by amikacin (80-85\%) and netilmicin (65$67 \%$ ). Susceptibility to gentamicin and ciprofloxacin was poor. In addition, a high level of ciprofloxacin resistance was seen among Salmonella paratyphi (90\%) and typhi (50\%) isolates [17].
Integrated surveillance of antimicrobial resistance and use in Colombo district

Having computerized data, extensive inter-connected surveillance networks, adequate laboratory capacity and rapid diagnostic facilities played a key role in the success of ESAC-Net and similar programmes established in resourcerich countries. In most of the resource-limited countries (RLCs), such resources are scarcely available. Hence, in order to assist RLCs to carry out efficient surveillance programmes, the WHO published a model for CommunityBased Surveillance of Antimicrobial Use and Resistance in Resource-Constrained Settings based on five pilot projects conducted in India [3] and South Africa [2, 14].

For surveillance of antibacterial resistance, the model recommends monitoring the resistance pattern of Escherichia coli (E. coli) isolated from urine samples of patients with urinary tract infections presented to public and private outpatient departments. For ABA use data, the model recommends collecting data from multiple facilities, both from the public and private sectors, from which people living in the geographical area might obtain ABA for ambulatory use. This model was piloted in the Colombo district.

In the public sector, of the 2183 urine samples, pathogenic E. coli was isolated in $9.3 \%(204)$, and $8 \%(n=16)$ of them were Extended Spectrum Beta Lactamase (ESBL) producers. E. coli was most resistant to ampicillin (85\%), followed by nalidixic acid (58.5\%), trimethoprim/sulphamethoxazole (47.1\%), ciprofloxacin (46.2\%), norfloxacin $(43.7 \%)$ amoxicillin /clavulanic acid (36.3\%), gentamicin (23\%) and nitrofurantoin (9\%). Multi-drug resistance was seen in $44 \%$ (18). In the private sector, of the 969 pathogenic E. coli, $28 \%$ were ESBL producers. E. coli was most resistant to ampicillin $(80 \%)$ followed by nalidixic acid $(67.8 \%)$, ciprofloxacin (59.5\%),norfloxacin(59\%),trimethoprim / sulphamethoxazole (52.5\%), amoxicillin /clavulanic acid (44\%), gentamicin (33\%) and nitrofurantoin (15\%) Multi-drug resistance was seen in 53\% (Senadeera et al, unpublished data).

Empirical use of ABAs with high resistance rates is known to be associated with an increased risk of treatment failure and selection of resistant strains. Surveillance of ABA use (Senadeera, et al, unpublished data) documented that of a total of 22321 prescriptions containing at least on ABA, amoxicillin /clavulanic acid accounted for $17.5 \%$ followed by amoxicillin (15\%), cefuroxime (11\%), azithromycin $(8 \%)$, doxycycline $(7.8 \%)$, clarithromycin $(7.1 \%)$, ciprofloxacin $(6.5 \%)$ and levofloxacin $(5.1 \%)$. From this data, one may predict that resistance to amoxicillin /clavulanic acid would also escalate similar to what has happened amoxicillin.

\section{Drivers for these initiatives}

These accelerated initiatives are mainly due to two reasons: Escalating problem of $\mathrm{ABR}$ and the broken antibiotic 
pipeline. Resistance to ABA is defined clinically as "a state in which a patient, when infected with a specific pathogen, is treated with an adequate antimicrobial dosage and administration schedule, but clinical criteria of cure (at a clinical and/or a microbiological level) are not reached" and microbiologically as "a state in which an isolate has a resistance mechanism rendering it less susceptible than to other members of the same species lacking any resistance mechanism" [19]. Alarming findings documented in a WHO global surveillance report published in 2014 inclde [20]:

1. Klebsiella pneumonia: Resistance to third-generation cephalosporin was $>30 \%$ in many countries and $>60 \%$ in some countries. Resistance to carbapenems exceeding $50 \%$ has reported

2. Escherichia coli: Resistance to fluoroquinolones and third generation cephalosporin $>50 \%$ in 5 out of $6 \mathrm{WHO}$ regions

3. MRSA resistance rates $>20 \%$ in all $\mathrm{WHO}$ regions and in some regions it is $>80 \%$

The report documented very high rates of $A B R$ in bacteria causing common health-care associated and communityacquired infections in all WHO regions and identified important gaps in existing surveillance programmes such as lack of data from countries with high disease rates and lack of standards for methodology.

Many pharmaceutical companies have abandoned the field of development of new ABA as it needs long time and huge investment. Only handful of the big pharmaceutical industries still continue to work on ABA development programmes, with others have closed the antibiotic research facility and manufacturing drugs that have proven sustainability in the market [9]. The three main factors responsible for pharmaceutical industries' lack of interest in antibiotics include:

1. Scientific: Most of the known drug targets have been already exploited.

2. Commercial: ABAs have limited prospects for profit as they are generally prescribed for short duration and most of the times cure the target disease. In addition, when the bacteria develop resistance, the demand for that ABA dramatically declines.

3. Regulatory bottlenecks: The regulatory requirements have become very demanding.

There is an urgent need to fix the broken antibiotic pipeline: In 2010, the Infectious Diseases Society of America launched the " $10 \times 20$ " initiative with the aim of producing 10 new antibiotics by 2020. In the first 6 years (2010-2016), though 6 new ABAs have received FDA approval, only one was a novel compound [21]. The WHO has published the global priority list of antibiotic-resistant bacteria to guide research, discovery and development of new ABAs [22]. Pathogens listed under critical priority are carbapenem-resistant Acinetobacter baumannii, Pseudomonas aeruginosa, and Enterobacteriaceae and third-generation cephalosporinresistant Enterobacteriaceae. Pathogens like clarithromycinresistant Helicobacter pylori, fluoroquinolone-resistant Salmonella spp., third-generation cephalosporin-resistant Neisseria gonorrhoea, and fluoroquinolone-resistant Neisseria gonorrhoea are listed under second priority level. Penicillin non-susceptible Streptococcus pneumonia is in the 3rd level.

\section{Antibacterial agents' stewardship programmes}

Antibacterial agents' stewardship programme (ASP) is a hospital-level programme committed to improve use of ABAs [23]. The CDC recommends that all acute-care hospitals to implement ASP because improving use of ABAs is an important patient safety and public health issue as well as a national priority $[11,23]$. Several benefits of such ASPs have been documented such as optimizing treatment of infections, reducing adverse effects associated with ABA use, improving quality of patient care, improving patient safety, increasing cure rates, reducing treatment failures, increasing correct prescribing and most importantly reduce ABR [2429]. The core elements of ASPs suggested by the CDC are [1] leadership commitment [2], accountability [3], drug expertise [4], action [5], tracking [6], reporting [7] and, education [23].

Problem of ABR had to be confronted seriously: the innumerous initiatives and publications available in the literature is testimony that no intervention is entirely satisfactory in combating the problem. As long as ABAs are in use, ABR is an inevitable consequence.

"While it is self-evident that the use of antimicrobial drugs has imposed selective pressures on the emergence of resistant microbes, to attribute the development of resistance entirely to imprudent antimicrobial use is, a fallacy that reflects an alarming lack of respect for the incredible power of microbes" [30].

All what we can do is to delay the emergence and spread of ABR by implementing these initiatives.

\section{References}

1. World Health Organization. The Selection of essential drugs. Report of the WHO Expert Committee. 2017 (including the 20th model list of Essential Medicines and 6th WHO model list of Essential Medicines for Children) Technical Report Series unedited. Geneva: WHO; 2017. Available from

http://www.who.int/medicines/publications/essentialmedicines /EML_2017_EC21_Unedited_Full_Report.pdf. accessed November 2017

2. World Health Organization. Essential Medicines and Health Products.

http://www.who.int/medicines/services/essmedicines_def/en/ accessed October 2017 
3. The Sri Lanka College of Microbiologists in collaboration with other professional colleges in healthcare and the Ministry of Healthcare, Nutrition and Indigenous Medicine. Empirical and prophylactic use of antimicrobials. National Guidelines. Colombo: SLCM; 2016. slmicrobiology.net/antibioticguidelines-2016. Accessed November 2017.

4. Hessen M T, Kaye D. Principles of use of antibacterial agents. Infect Dis Clin NAm 2004; 18: 435-450.

https://doi.org/10.1016/j.idc.2004.04.002

5. National Institute for Health and Care Excellence. Surgical site infections: prevention and treatment. United Kingdom: NICE; 2008. https://www.nice.org.uk/guidance/cg74/ resources /surgical-site-infections-prevention-and-treatment-pdf975628422853

6. World Health Organization. Global guidelines for the prevention of surgical site infections. Geneva: WHO; 2016

7. Berríos-Torres SI, Umscheid CA, Bratzler DW, et al. for the Healthcare Infection Control Practices Advisory Committee. Center for Disease Control and Prevention Guideline for the Prevention of Surgical Site Infection, 2017. JAMA Surg. 2017; 152(8):784-791.https://doi.org/10.1001/jamasurg.2017.0904

8. Chang-Ro Lee CR, Cho IH, Jeong BC, Lee SH. Strategies to minimize antibiotic resistance. Int. J. Environ. Res. Public Health 2013;10: 4274-305. https://doi.org/10.3390/ijerph10094274

9. Carlet J, Jarlier V, Harbarth S, et al. Participants of the 3rd World Healthcare-Associated Infections Forum. Ready for a world without antibiotics? The Pensières Antibiotic Resistance Call to Action. Antimicrob Resist Infect Control 2012;1:11 https://doi.org/10.1186/2047-2994-1-11

10. World Health Organization. The evolving threat of antimicrobial resistance: options for action. Geneva: WHO; 2012

11.Centres for Disease Control and Prevention. Antibiotic Resistance Threats in the United States, 2013. Atlanta, Georgia: U.S. Department of Health and Human Services, CDC; 2013. https://www.cdc.gov/drugresistance/pdf/ar-threats-2013508.pdf. Accessed November 2017

12. World Health Organization. Critically important antimicrobials for human medicine. 5th revision, 2016. Geneva: WHO; 2017. http://apps.who.int/iris /bitstream /10665/251715/1/97892415 11469-eng.pdf?ua=1. Accessed October 2017

13.Ministry of Healthcare, Nutrition and Indigenous Medicine. National Strategic Plan for Combating Antimicrobial Resistance in Sri Lanka 2017 - 2022. 1st edition; 2017.

http://www.searo.who.int/srilanka/areas/antimicrobial_resistanc e/national_strategy_amr.pdf?ua=1. Accessed November 2017

14.Holloway K, Mathai E, Sorensen T, Gray A. Community based surveillance of antimicrobial use and resistance in resourceconstrained settings. Geneva: World Health Organization, 2009

15. Weist K, Högberg LD. ECDC publishes 2015 surveillance data on antimicrobial resistance and antimicrobial consumption in Europe. Eurosurveillance. 2016;21(46):30401.

https://doi.org/10.2807/1560-7917.ES.2016.21.46.30399

16. Jayatilleke SK, Patabendige G. Dassanayake M. et al. Analysis of urine culture isolates from seven laboratories in Western province of Sri Lanka. National Laboratory Based Surveillance- conducted by the Sri Lanka College of Microbiologists -2014. Sri Lankan Journal of Infectious Diseases 2016 Vol.6 (1):17-24. DOI: http://dx.doi.org/10.4038/sljid.v6i1.8105
17.ARSP Working Group, The Sri Lanka College of Microbiologists. A multi centre laboratory study of Gram negative bacterial blood stream infections in Sri Lanka. Ceylon Medical Journal 2013; 58: 56-61. https://doi.org/10.4038/cmj.v58i2.5680

18.Senadeera GPSG, Sri Ranganathan S, Patabendige G, et al. Resistance and utilisation pattern of antibacterial agents in outpatient settings in two Teaching Hospitals in Colombo. Ceylon Medical Journal 2016; 61:113-117:

http://doi.org/10.4038/cmj.v61i3.8346

19.Canto' n R, Morosini MI. Emergence and spread of antibiotic resistance following exposure to Antibiotics. FEMS Microbiol Rev 35; 2011: 977-991.https://doi.org/10.1111/j.15746976.2011.00295.x

20.World Health Organization. Antimicrobial Resistance. Global report on Surveillance. Geneva: WHO; 2014

21.Boucher HW, Talbot GH, Benjamin DK, et al. $10 \times$ '20 Progress Development of New Drugs Active Against Gram-Negative Bacilli: An Update From the Infectious Diseases Society of America. Clin Infect Dis 2013; 56: 1685-94.

https://doi.org/10.1093/cid/cit152

22.World Health Organization. Global priority list of antibioticresistant bacteria to guide research, discovery, and development of new antibiotics. Geneva: WHO; 2015

23.CDC. Core Elements of Hospital Antibiotic Stewardship Programs. Atlanta, GA: US Department of Health and Human Services, CDC;2014. Available at http://www.cdc.gov/getsmart/ healthcare/ implementation/core-elements.html

24.Davey P, Brown E, Charani E, et al. Interventions to improve antibiotic prescribing practices for hospital inpatients. The Cochrane database of systematic reviews. 2013;4:CD003543. https://doi.org/10.1002/14651858.CD003543.pub3

25.Malani AN, Richards PG, Kapila S, Otto MH, Czerwinski J, Singal B. Clinical and economic outcomes from a community hospital's antimicrobial stewardship program. American journal of infection control. Feb 2013; 41(2):145-148.

https://doi.org/10.1016/j.ajic.2012.02.021

26.Stach LM, Hedican EB, Herigon JC, Jackson MA, Newland JG. Clinicians' Attitudes Towards an Antimicrobial Stewardship Program at a Children's Hospital. Journal of the Pediatric Infectious Diseases Society. September 1, 2012 2012; 1(3): 190-197.

27.Khaki R, Elligsen M, Walker S, Simor A, Palmay L, Daneman N. Impact of antimicrobial stewardship in critical care: a systematic review. The Journal of antimicrobial chemotherapy. Jun 2011;66(6):1223-1230. https://doi.org/10.1093/jac/dkr137

28. Valiquette L, Cossette B, Garant MP, Diab H, Pepin J. Impact of a reduction in the use of high-risk antibiotics on the course of an epidemic of Clostridium difficile-associated disease caused by the hypervirulent NAP1/027 strain. Clinical infectious diseases : an official publication of the Infectious Diseases Society of America. Sep 1 2007;45 Suppl2:S112-121. https://doi.org/10.1086/519258

29.DiazGranados CA. Prospective audit for antimicrobial stewardship in intensive care: impact on resistance and clinical outcomes. American journal of infection control. Aug 2012;40(6):526-529. https://doi.org/10.1016/j.ajic.2011.07.011

30.Spellberg B, Guidos R, Gilbert D, et al. The epidemic of antibiotic-resistant infections: a call to action for the medical community from the Infectious Diseases Society of America. Clin Infect Dis 2008;46:155-64. https://doi.org/10.1086/524891 